\title{
Frequency Diverse Array Antennas: From Their Origin to Their Application in Wireless Communication Systems
}

\author{
Shaddrack Yaw Nusenu $\mathbb{I D}^{1,2}$ and Abdul Basit $\mathbb{D}^{1,3}$ \\ ${ }^{1}$ University of Electronic Science and Technology of China, Chengdu, China \\ ${ }^{2}$ Koforidua Technical University (KTU), Koforidua, Ghana \\ ${ }^{3}$ Department of Electrical Engineering, International Islamic University, Islamabad, Pakistan \\ Correspondence should be addressed to Shaddrack Yaw Nusenu; nusenu2012gh@yahoo.com
}

Received 10 December 2017; Accepted 29 March 2018; Published 8 May 2018

Academic Editor: Gianluigi Ferrari

Copyright (c) 2018 Shaddrack Yaw Nusenu and Abdul Basit. This is an open access article distributed under the Creative Commons Attribution License, which permits unrestricted use, distribution, and reproduction in any medium, provided the original work is properly cited.

\begin{abstract}
Wireless communication systems have gained considerable growth rate nowadays, with the anticipation that communications will be available everywhere and anywhere in the near future. Phased array antenna whose beam steering is fixed in an angle for all range cells has been utilized for wireless communications. To mitigate this problem, a new array concept, namely, frequency diverse array (FDA), was proposed. This paper presents how FDA technology could be useful in today's wireless communication technology. FDA is distinct from phased array in a sense that it employs frequency increment across array elements. The use of a frequency increment creates a beam steering that is a function of angle, time, and range which allows the FDA antenna to transmit the energy along the prespecified range and angle direction. In addition, we consider the time-variant beampattern aspect of an FDA, which has normally been ignored in the literature. In this study, we present the mathematical fundamentals of FDA antenna and why it could be exploited for wireless communication systems. Furthermore, FDA using Butler matrix for communication has been discussed. Performance analysis in terms of transmit beampattern, signal-to-interference-and-noise ratio (SINR), and direction of arrival has been presented and compared with that of phased array antenna.
\end{abstract}

\section{Introduction}

Wireless communication systems have become very promising than their counterpart wired communication due to their merits [1], and this has attracted a huge demand on wireless technology, hence the explosive rate in many technical fields. The proposed fifth generation (5G) of wireless cellular communication systems is envisaged to be available by 2020 [2]. Besides, antenna array applications have been put forward in recent years for wireless communications systems. The array antennas' advantages can be summarized as (1) increasing channel capacity and spectrum efficiency, (2) extending range coverage, (3) tailoring beam shape, (4) steering multiple beams to track many mobiles, and (5) compensating aperture distortion electronically. In addition, they reduce multipath fading and cancel cochannel interferences, BER, and outage probability $[3,4]$.

An array of antennas works on the principle that the desired signal and unwanted signal (cochannel interferences) arrive from diverse directions. Therefore, the beampattern could be adjusted by combining signals from the array antennas with appropriate weighting factor to suit the requirements. Antennas can be classified as omnidirectional, directional, phased array, and frequency diverse array. Phased array antennas have been employed in many applications, for example, in radar systems, electronic warfare, radio astronomy, and airport safety and communications [5]. Phased array antennas have the capability to steer the beam electronically with high directivity. The offered directional gain is beneficial for detecting mobile users, tracking users, and suppressing sidelobe interferences from other directions [6]. Although phased arrays have several advantages, some of their drawbacks in many applications are as follows: (1) One of the major disadvantage is their high manufacturing cost. A more directed beam requires a greater number of antenna elements and electronic phase shifters, which are quite expensive [7]. (2) In phased arrays, exceeding interelement distance beyond half wavelength results in the appearance of 
grating lobes in the visible regions that deteriorates the communication user(s) tracking performance [8]. (3) Phased array antenna produces a power maximum at a fixed angle for all ranges. Therefore, to suppress range-dependent interferences and to localize multiple targets with the same direction but distinct ranges using phased arrays is very difficult.

In order to circumvent this problem, recently, a flexible beam scanning array, namely, frequency diverse array (FDA), was proposed by $[9,10]$ to overcome the limitation of beam steering which is fixed at an angle for all the range cells [6] in phased array and FDA has no phase shifters. In FDA, a small frequency increment is applied across the array elements, and this frequency increment results in a range-angle-dependent beampattern [9]. The FDA is quite different from multipleinput multiple-output (MIMO) [11] radar as it transmits overlapped signals closely spaced in frequencies, while MIMO, in some cases, employs orthogonal signals from widely separated antennas (i.e., widely spaced MIMO) over multiple independent paths to provide spatial diversity [12] and in some cases, uses multiple waveforms to provide waveform diversity from closely located antennas (i.e., colocated MIMO) [13]. Likewise, FDA is also different from orthogonal frequency division multiplexing (OFDM) [14]. The latter uses orthogonal subcarriers [15]. Moreover, an FDA is also different from the conventional frequency scanning arrays [16], where each element uses the same frequency at a given time [17]. Different from phased arrays, the FDA beampattern provides a global maximum, as well as, a number of local maxima at diverse angle and ranges values [18]. This ability can be utilized for detecting multiple targets having same directions, but different range values. Note that the introduction of frequency offsets, the apparent scan angle, is not equal to its nominal scan angle as well as the actual beam-steering direction cannot be effectively predicted as phased arrays. Furthermore, since the phases of the FDA transmitted signals add constructively in certain regions, while destructively in others, this property can be utilized to suppress interferences or avoid transmitting the signals to some undesired regions [19]. In [20-23], discussed about the applications of FDA and recently, FDA antennas have been utilized in wireless communications for security applications [14, 24-28].

In this paper, we give a review (i.e., origin) on the development of FDA antenna technology that is from phased array antenna to FDA antenna. We also highlight the potential applications of using FDA in communication areas. Additionally, we appeal to the antenna and wireless communication research communities to contribute in terms of more publications on FDA antenna research and development. Performance analysis in terms of transmit beampattern, SINR, and direction of arriving is then evaluated, and we give example of FDA using $4 \times 4$ Butler matrix for communication application.

The rest of this paper is organized as follows: Section 2 presents an overview and some necessary details about the data model of frequency diverse array (FDA) antenna. Section 3 explains why the FDA antenna could be used for wireless communication systems. Section 4 presents FDA antenna architecture schemes. Section 5 presents FDA and Butler matrix scheme for communication. Next, performance

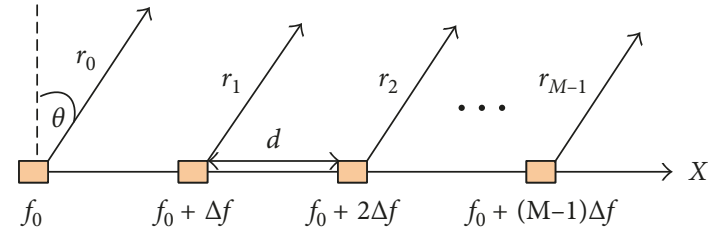

Figure 1: Illustration of linear Frequency diverse array.

analysis is presented in Section 6, followed by numerical results in Section 7. Finally, concluding summaries are drawn in Section 8.

\section{Mathematical Formulation of FDA Antenna}

In a standard phased array, it is assumed that identical transmitted signal is emitted from the individual array element, excluding the amplitudes and phases. The FDA antenna, which is different from phased arrays, can be excited by the same signal or different signals. For simplicity and without loss of validity, we assume that the waveforms radiated from each FDA antenna element are identical with a frequency increment or offsets $\Delta f$ and element spacing $d$.

Consider a number of FDA antenna elements, $M$, as shown in Figure 1, and the radiation frequency of each element can be given by [19].

$$
f_{m}=f_{0}+(m-1) \cdot \Delta f, \quad m=1,2,3, \ldots, M,
$$

where $f_{0}$ denotes the radar operating carrier frequency.

And the phase of the signal transmitted by the first antenna element can be written as

$$
\phi_{1}=\frac{2 \pi f_{0} r_{1}}{c},
$$

where $c$ is the speed of light, $r_{1}$ denotes the distance between the first element and the observed point target. Similarly, the phase of the signal transmitted by the second element can be expressed as

$$
\phi_{2}=\frac{2 \pi f_{2} r_{2}}{c}=\frac{2 \pi\left(f_{0}+\Delta f\right) r_{2}}{c} .
$$

The phase difference between the signals arriving at the first and second elements, respectively, is given in (4)

$$
\Delta \phi_{1}=\phi_{2}-\phi_{1}=-\frac{2 \pi f_{0} d \sin \theta}{c}-\frac{2 \pi \Delta f d \sin \theta}{c}+\frac{2 \pi \Delta f r_{1}}{c} .
$$

Similarly, (5) is the phase difference between the first and the $m$ th element;

$$
\begin{aligned}
\Delta \phi_{m-1}=\phi_{m}-\phi_{1}= & -\frac{2 \pi f_{0}(m-1) d \sin \theta}{c}+\frac{2 \pi(m-1) \Delta f r_{1}}{c} \\
& -\frac{2 \pi(m-1)^{2} \Delta f d \sin \theta}{c},
\end{aligned}
$$

where $\theta$ denotes the angle direction of the target, $r_{m} \approx r-(m-1) d \sin \theta$ is the difference between individual elements approximation with $r=r_{1}$ being the slant range of 


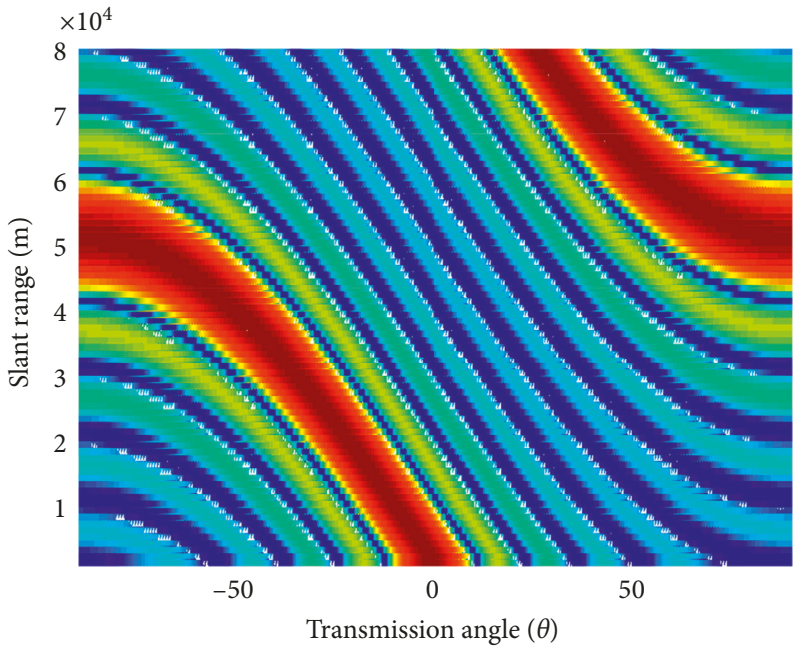

(a)

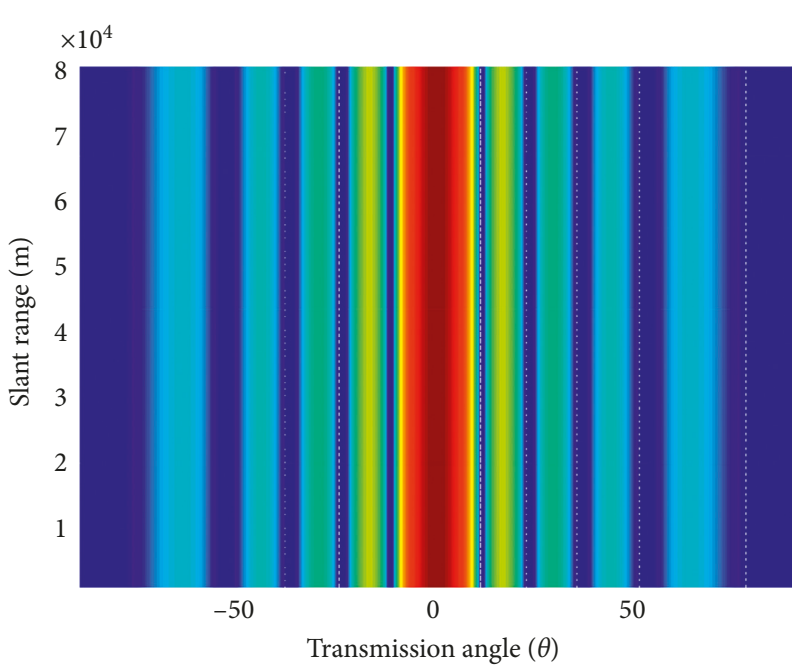

(b)

Figure 2: Transmit beampattern: (a) conventional FDA antenna and (b) phased array antenna.

the first element. Note that the first term in (5) is simply the conventional array factor seen in the array theory. The last term is of significance since it shows that the radiation beampattern of the array depends on both the range and frequency increment. It should be noted that the FDA antenna is different from conventional frequency scanning arrays. Frequency scanned arrays employ the frequency increment as a function of time for all elements, while the FDA antenna applies the frequency increment as a function of the elements.

Taking the first element as the reference for the array, the steering vector of the FDA antenna is expressed as in (6), with $r$ denoting the slant range of the first element,

$$
\mathbf{a}(\theta, r, t)=\mathbf{a}_{\theta}(\theta) \odot \mathbf{a}_{r}(r) \odot \mathbf{a}_{t}(t),
$$

where

$$
\begin{aligned}
& \mathbf{a}_{\theta}(\theta)=\left(1, \exp \left(\frac{j 2 \pi m d \sin \theta}{\lambda}\right), \ldots, \exp \left(\frac{j 2 \pi M d \sin \theta}{\lambda}\right)\right)^{T}, \\
& \mathbf{a}_{r}(r)=\left(1, \exp \left(\frac{j 2 \pi \Delta f r}{c}\right), \ldots, \exp \left(\frac{j 2 \pi M \Delta f r}{c}\right)\right)^{T}, \\
& \mathbf{a}_{t}(t)=(1, \exp (j 2 \pi \Delta f t), \ldots, \exp (j 2 \pi M \Delta f t))^{T},
\end{aligned}
$$

with $\odot$ denoting the Hadamard product and $(\cdot)^{T}$ being the transpose operator.

The following summarized FDA antenna characteristics $[19,21]$ :

(1) If the frequency increment $\Delta f$ is fixed, the beam direction is a function of range $r$ dependent.

(2) If the range $r$ is fixed, the beam direction is a function of $\Delta f$ dependent.

(3) If the frequency increment $\Delta f$ is not employed $(\Delta f=0)$, it simplified to phased array antenna.

(4) As the frequency increment $\Delta f$ influences the beamwidth, a higher resolution may be achieved for the FDA as compared to the phased array antenna.
Hence, the FDA antenna is rather different from phased arrays. It is a new array concept and novel beam scanning technique. The range-dependent and frequency offset-dependent beamforming is of great importance because it can achieve local maxima at different ranges as this may provide many promising application potentials in wireless communication systems in future.

Assume the following parameters: $M=10, \Delta f=30 \mathrm{kHz}$, $f_{0}=10 \mathrm{GHz}$, and $d=(1 / 2) \lambda$, in Figure 2 , we show the transmit beampattern of the conventional FDA antenna, and phased array antenna.

It can be noticed that in Figure 2(a), the conventional FDA yields range-angle-dependent beampattern, and Figure 2(b) depicts the phased array antenna which yields only angledependent beampattern with no range-dependent capability.

\section{Why FDA Could Be Used for Wireless Communication Systems}

According [19], the maximum field can be obtained as

$$
\Delta f t-\frac{\Delta f r}{c}+\frac{d f_{0} \sin \theta}{c}+\frac{\Delta f d \sin \theta}{c}=z, \quad z=0, \pm 1, \ldots
$$

From (8), it is clear that there are multiple solutions for the unfixed variables, when only one variable is fixed. Moreover, when two variables are fixed, the periodicity array pattern depends on the unfixed variable. For instance, if $(8)$ is solved for the following variables $t, r$, and $\theta$, we can express as follows:

$$
\begin{aligned}
& t=\frac{z}{\Delta f}+\frac{r}{c}-\frac{d f_{0} \sin \theta}{c f_{0} \Delta f}+\frac{d \sin \theta}{c}, \\
& r=c t+\frac{d f_{0} \sin \theta}{\Delta f}-\frac{z c}{\Delta f}+d \sin \theta, \\
& \theta=\sin ^{-1}\left[\frac{z c-\Delta f(c t-r)}{d f_{0}+\Delta f d}\right] .
\end{aligned}
$$




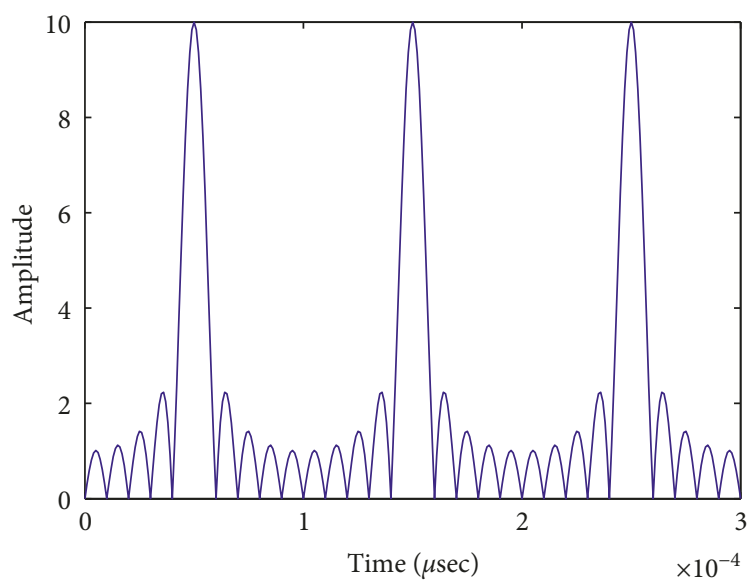

(a)

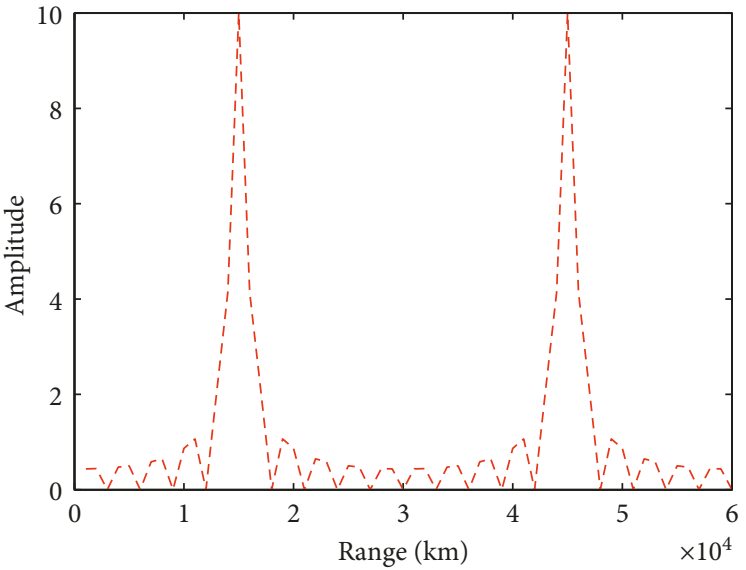

(b)

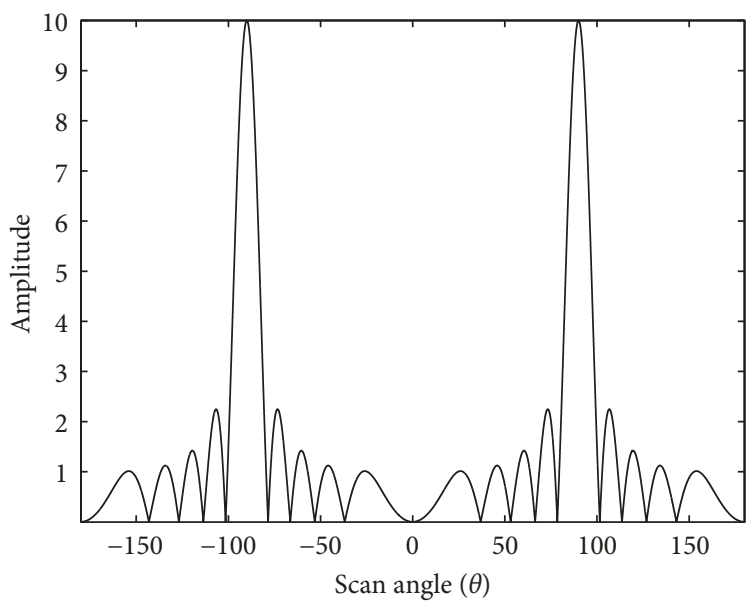

(c)

Figure 3: Array factor periodicity of FDA in (a) time dimension, (b) range dimension, and (c) scan angle dimension.

Figure 3 illustrates the array factor periodicity of FDA antenna using (9).

Now it is evident that FDA antenna has autoscanning property [29] which is a unique property that could be exploited for wireless communication systems application, for instance at the base station and communication devices.

3.1. Range and Angle Estimation of Mobile Targets/Users. As noted already, FDA provides range-dependent beampattern. Therefore, it has the capability of suppressing rangedependent interferences. For instance, Figure 4 shows comparative transmit beampattern in suppressing rangedependent interferences. It can be noticed that in Figure 4(a), the FDA beampattern can identify the target $\left(30 \mathrm{~km}, 40^{\circ}\right)$ and suppress the interferences, for example, $\left(50 \mathrm{~km}, 40^{\circ}\right)$ and $\left(20 \mathrm{~km}, 40^{\circ}\right)$, effectively due to the frequency offsets employed, whereas the phased array beampattern (Figure 4(b)) cannot effectively identify the target and interferences. Nevertheless, due to coupling range and angle peaks in the beamformer output, FDA antenna cannot estimate the range and angle of a target solely. It should be noted that the range-angle response can be decoupled as depicted in Figure 5.

3.2. Security for Wireless Communication Systems. One of the major issues in wireless communication systems is security, because of the broadcasting nature of wireless signals. Therefore, any receiver is able to have a copy of the transmitted signal, making the activities of the eavesdroppers much easier compared to wired communication [32]. The work done by Wyner [33] created the concept of physical-layer security. The fundamental principle behind physical-layer security is to exploit the normal randomness of wireless communication channels to ensure that the information to the desired destination is secured, and at the same time, this information cannot be extracted by an undesired receiver [34]. Physical-layer security can be implemented by employing various techniques [35] such as beam-steering and jamming. In beam-steering techniques, the transmitter steers the mainlobe along the desired direction, and steer the null towards the undesired direction [36-38]. As stated before, by employing the frequency offsets, the apparent scan angle is not equal to its nominal 


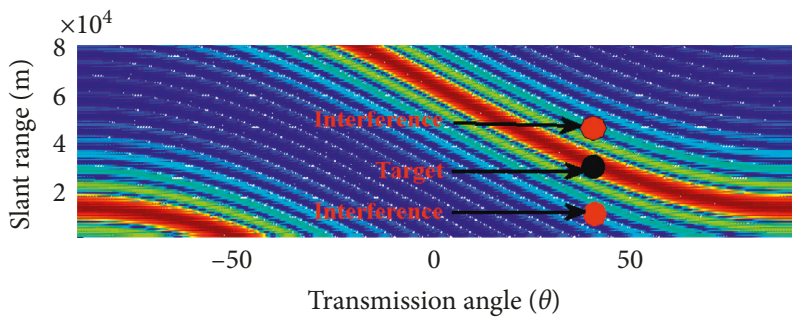

(a)

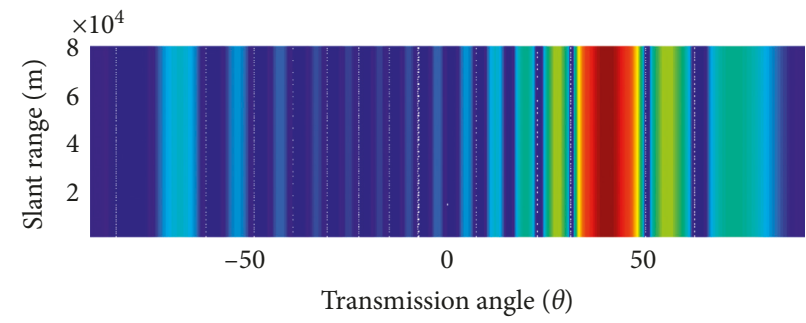

(b)

FIGURE 4: Comparative performance of transmit beampattern in suppressing range-dependent interferences: (a) FDA antenna (top) and (b) phased array antenna (bottom).

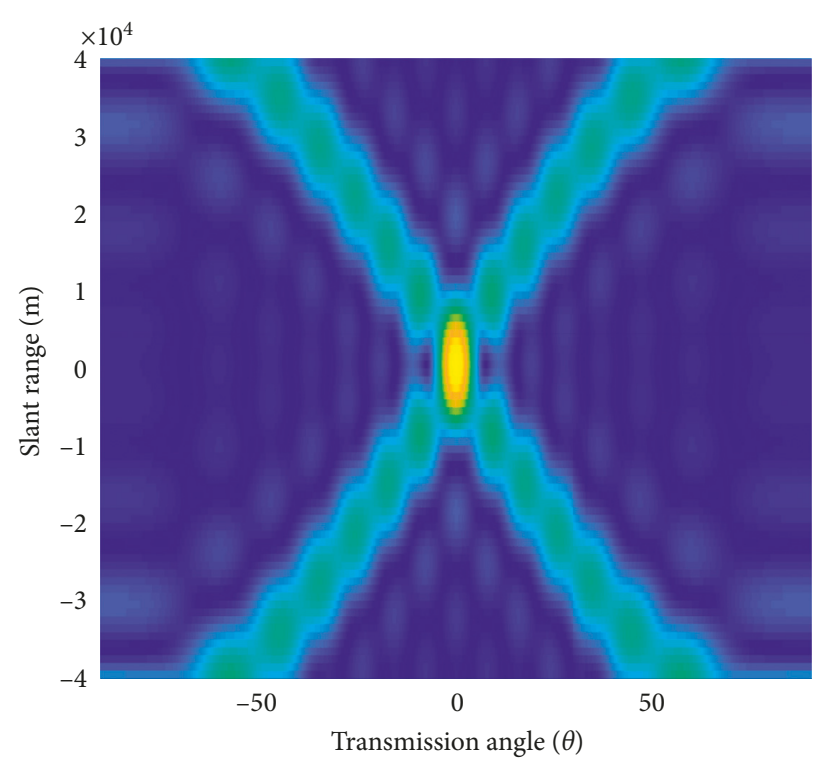

(a)

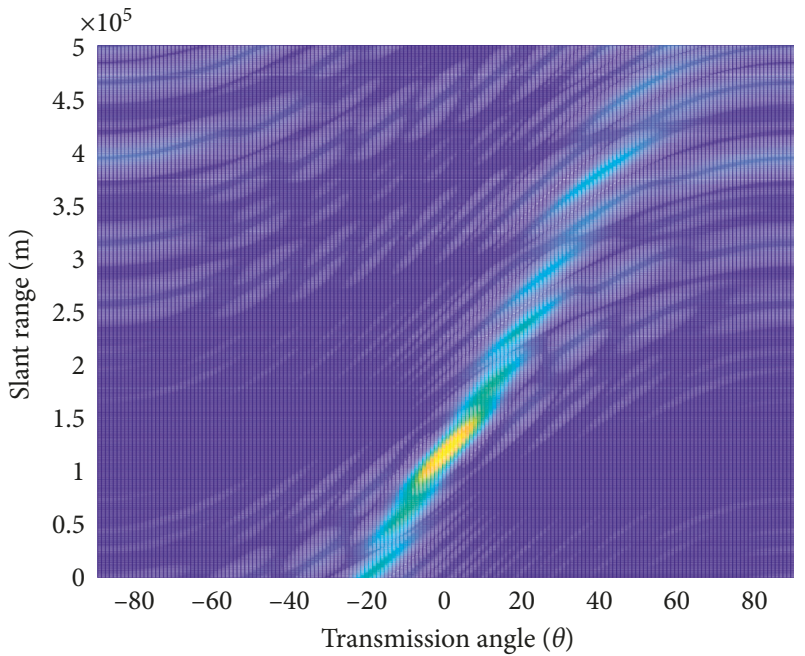

(b)

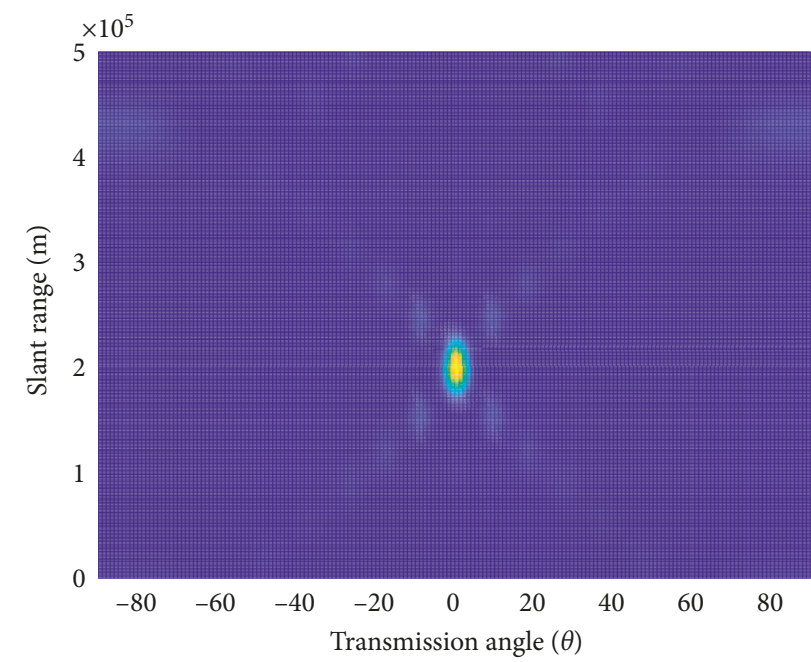

(c)

FIgURE 5: Comparison of the beampattern generated by (a) symmetrical FDA [19, 28], (b) log FDA [30], and (c) hamming FDA [31].

scan angle as well as the actual beam-steering direction cannot be effectively predicted as phased array antenna; hence, this property can be utilized to suppress interferences, avoid transmitting the signals to some undesired regions, and design low probability of interception required for physical-layer security in wireless communication systems [27]. Figure 6 shows the illustration of FDA antenna's apparent direction angle and physical direction angle. 


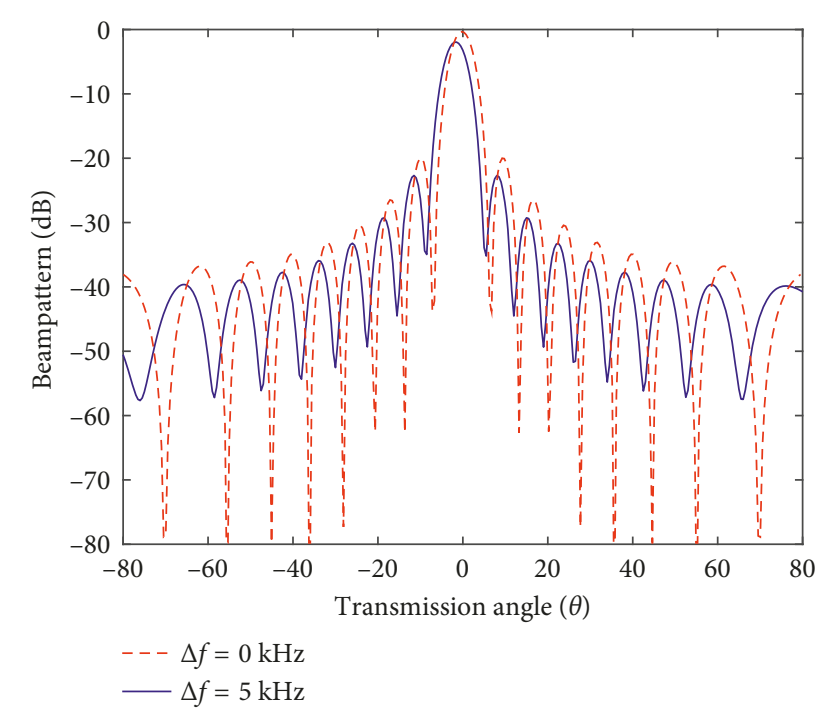

FIGURE 6: FDA antenna apparent direction angle and physical direction angle.

\section{FDA Antenna Architecture Schemes}

4.1. FDA Antenna Architecture. In the FDA antenna, we have uniform amplitude weightings and/or nonuniform weightings and uniform frequency offsets and nonuniform frequency offset. The antenna sidelobes can be decreased by

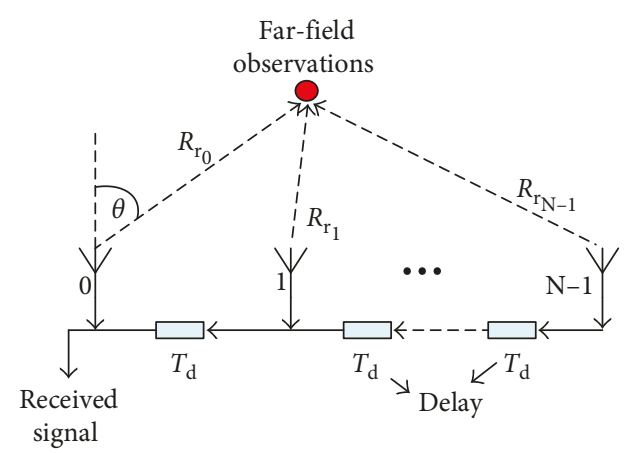

FIGURE 7: FDA antenna receiving architecture.

applying nonuniform weightings but at the expense of the beamwidth. At the transmitting side, the far-field of the FDA beampattern can be expressed as [39, 40],

$$
\mathrm{FDA}_{T}=\sum_{m=1}^{M} \frac{a_{m}}{r_{m}} f_{\mathrm{e}}\left(f_{m}\right) \exp \left\{j 2 \pi f_{m} t-j \frac{2 \pi f_{m} r_{m}}{c}-j m \phi\right\},
$$

where $a_{m}$ is the transmitting weightings and $\phi$ denotes the phase difference between two elements.

At the receiving side, suppose the incoming signal is a continuous signal, $s(t)=\exp \left(j 2 \pi f_{0} t\right)$, then FDA far-field array pattern is expressed as in (11) [40].

$$
\begin{aligned}
\mathrm{FDA}_{R} & =\sum_{n=1}^{N} \frac{a_{n}}{R_{r_{n}}} s\left(t-\tau_{n}\right) \exp \left\{-j \frac{2 \pi f_{0}\left(t-\tau_{n}\right)}{c} R_{r_{n}}\right\} \exp \left(j 2 \pi \frac{v_{r} f_{0}}{c} t\right) \\
& \simeq \frac{a_{n}}{R_{r_{0}}} \sum_{n=1}^{N} \exp \left\{j n\left(-2 \pi f_{0} T_{d}+\frac{2 \pi f_{0} d \sin \theta}{c}\right)\right\} \exp \left\{j\left(2 \pi f_{0} t-\frac{2 \pi f_{0} R_{r_{0}}}{c}\right)\right\} \exp \left(j 2 \pi \frac{v_{r} f_{0}}{c} t\right),
\end{aligned}
$$

where $a_{n}$ is the receiving weightings, $\tau_{n}=n T_{\mathrm{d}}$, where $T_{\mathrm{d}}$ is the interelement delay. It should be noted that, in communication systems, two moving platforms with relative velocity $v_{\mathrm{r}}$, the Doppler frequency shift $f_{\mathrm{d}}$ at the receiver is $f_{\mathrm{d}}=v_{\mathrm{r}} f_{0} / c$. Note that herein, equal Doppler frequency shift is adopted for the individual array element. Based on approximation, the distance from the far-field observation point to the $n$th array element is $R_{r_{n}} \simeq R_{r_{0}}-n d \sin \theta$ with $R_{r_{0}}$ denoting the distance from the point to the first receiving element. Also in amplitude sense, we can have $R_{r_{n}} \simeq R_{r_{0}}$. Figure 7 shows the FDA antenna receiving architecture.

By inspection, it can be seen that, similar to the transmitting FDA antenna (10), the receiving FDA antenna (11) also creates a range-angle-dependent beampattern. This implies that at the receiver, beamforming range-dependent nulls are possible. It should be noted that in the FDA antenna, changing the carrier frequency and frequency increment, every point in space can be scanned in a discrete way. Moreover, if the total frequency increments are increased, some points in space can be scanned with more than one frequency component and this diversity has advantages in terms of multipath interferences.
From the literature [41], we can jointly employ transmitting FDA and receiving FDA antennas. In addition, the concepts of combining transmitting FDA antenna and omnidirectional receiving antenna or omnidirectional transmitting antenna and receiving FDA antenna are also possible implementation. Table 1, compares the FDA antenna and phased array antenna. And Table 2, compares distinct FDA antenna configuration.

4.2. Adaptive/Optimal FDA Antenna Architecture. Figure 8 depicts adaptive FDA antenna architecture whereby the gain and the phase of the signals which are induced at each array element are altered before combining in order to adjust the gain of the array in a dynamic fashion, as the system required. The adaptive system provides a way for the antenna arrays to adapt to the situation, and the adaption process is generally influenced by the control of the system. On the other hand, an optimal FDA antenna can be exploited in such a way that the gain and phase of each antenna element are adjusted to provide an optimal performance. For instance, maximum output signal-to-interference-and-noise ratio (SINR) can be 
TABle 1: Antenna array comparisons: FDA and phased array.

\begin{tabular}{lccc}
\hline Antenna array & Transmitted signals & Gain (array) & Transmit capabilities \\
\hline FDA & Coherent & Yes & Range-angle-dependent \\
Phased array & Coherent & Yes & Angle-dependent only \\
\hline
\end{tabular}

TABle 2: Comparison among distinct FDA antenna configuration.

\begin{tabular}{|c|c|c|c|c|}
\hline Antenna configuration & Range and angle & $\begin{array}{l}\text { Computation } \\
\text { complexity }\end{array}$ & $\begin{array}{l}\text { Interference } \\
\text { suppression }\end{array}$ & $\begin{array}{l}\text { User detection } \\
\text { capability }\end{array}$ \\
\hline Conventional FDA & Coupled beampattern & Reasonable & Good & Good \\
\hline FDA with nonuniform frequency offset & $\begin{array}{c}\text { Decoupled } \\
\text { beampattern }\end{array}$ & Less & Improved & Improved \\
\hline
\end{tabular}

obtained by canceling unwanted interferences and receiving the desired signal without distortion by adjusting the gains and phases of each FDA antenna element.

\section{FDA Transmitter Scheme Using Butler Matrix for Wireless Communication}

Figure 9 shows a block diagram of two-dimensional (2-D) (i.e., range and angle) half wavelength spaced FDA antenna using a $4 \times 4$ Butler matrix [42-44] for wireless communication. We employ four FDA elements with carrier frequencies connected at the outport of a $4 \times 4$ Butler matrix, namely, $f_{0}, f_{0}+\Delta f, f_{0}+2 \Delta f$, and $f_{0}+3 \Delta f$, respectively, where $f_{0}$ is the carrier frequency and $\Delta f$ being the frequency increment across the array element.

The basic principle is that, when Port 1 is excited, it goes through the signal path, A-B-C-D to $f_{0}$, with phase shift of $135^{\circ}$. Similarly, a phase shift of $90^{\circ}$ is obtained between Port 1 and $f_{0}+\Delta f$ using the signal path A-B-C-E and so forth. Note that, since we employ a $4 \times 4$ Butler matrix, the FDA elements has been set to four. The phase differences of a $4 \times 4$ Butler matrix are $\pm 45^{\circ}$ for the output ports 1 and 4 , and $\pm 135^{\circ}$ for the output ports 2 and 3, respectively [45].

Here, we adopt a uniform linear frequency offset FDA antenna as depicted in Figure 1. The transfer function from

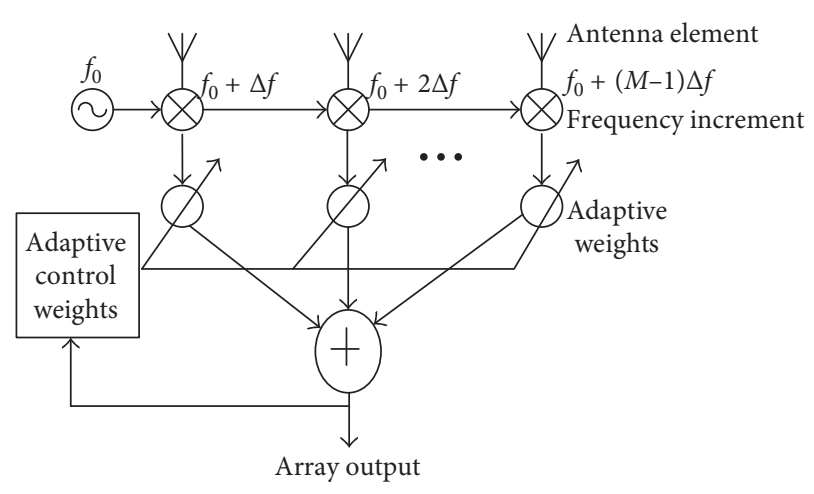

FIgURe 8: Adaptive FDA antenna architecture.

the $n$th input port to the $m$ th output port of the $4 \times 4$ Butler matrix is given as [46].

$$
\begin{aligned}
T_{m n}= & \frac{1}{\sqrt{N}} e^{-j(2 \pi / N)(m-(N+1 / 2))(n-(N+1 / 2))}, \\
& m, n=1,2, \ldots, N
\end{aligned}
$$

And the far-field radiation pattern of the $n$th Butler matrix input port excitation can be obtained by (13).

$$
F_{n}(\theta, r)=\sum_{m=1}^{N}\left[\left(\frac{1}{\sqrt{N}} e^{-j(2 \pi / N)(m-(N+1 / 2))(n-(N+1 / 2))}\right) e^{j\left[(m-(N+1 / 2)) \pi \sin (\theta)-\left(2 \pi \Delta f r / c_{0}\right)\right]}\right]
$$

This implies that the main beam pointing direction depends on not only the angle $\theta$ but also the range $r$ and frequency increment $\Delta f$. From (13), the beampattern peak corresponds to $\frac{2 \pi}{N}\left(m-\frac{N+1}{2}\right)\left(n-\frac{N+1}{2}\right)=\pi\left[\left(m-\frac{N+1}{2}\right) \sin \theta-\frac{2 \Delta f r}{c_{0}}\right]$.

And the corresponding instantaneous main beam pointing direction angle $\theta_{n}$ can be calculated by

$$
\theta_{n}=\sin ^{-1}\left[\frac{2 n-N-1}{N}+\frac{4 \Delta f r_{n}}{c_{0}(2 m-N-1)}\right] \text {. }
$$

Therefore, the instantaneous main beam pointing direction angle $\theta_{n}$ depends on both the range $r_{n}$ and the frequency increment $\Delta f$, which characteristics can be exploited for directional secure communications.

Similarly, by replacing $n$ in (13) and (15), with $q$, the farfield $F_{q}(\theta, r)$ generated by the $q$ th Butler matrix input port excitation with the instantaneous main beam pointing direction angle $\theta_{q}$ can be acquired. It is easily verified that $F_{n}\left(\theta_{q}\right)=0$ when $n \neq q$. This implies that $F_{q}(\theta, r)$ 's main beam is projected along the null radiation direction of $F_{n}(\theta, r)$. It should be noted that because of beam orthogonality property of a Butler matrix, we can have $F_{n}(\theta, r)$ 's main beam and $F_{q}(\theta, r)$ 's null direction. 


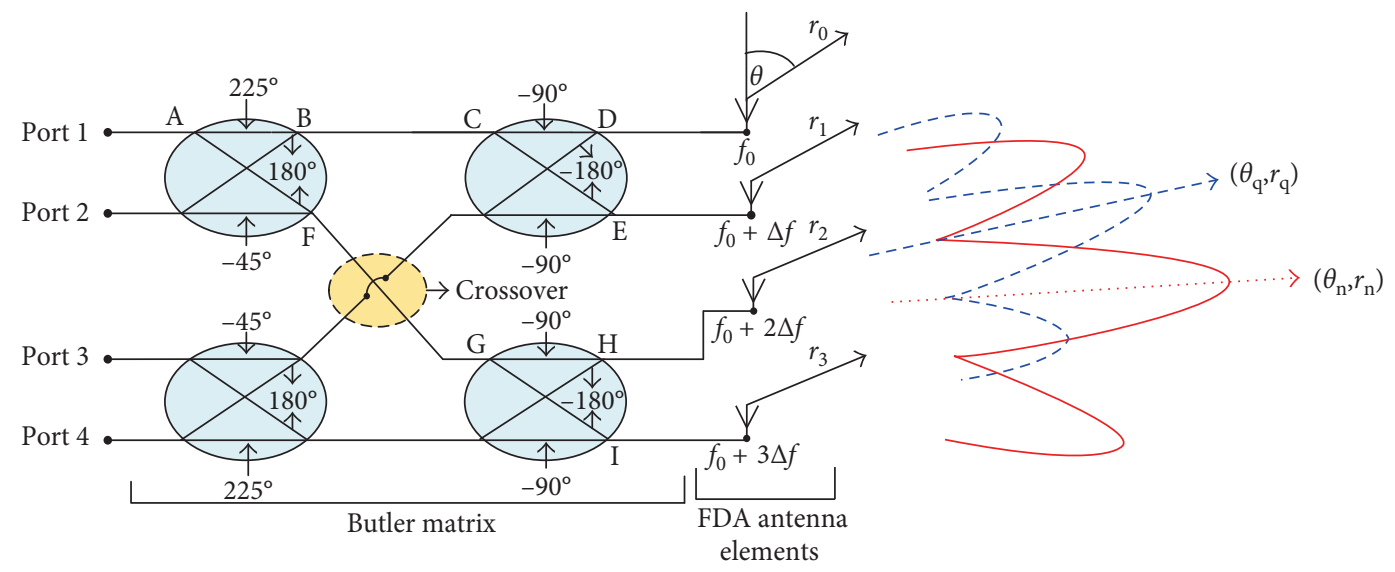

FIgURE 9: FDA using a $4 \times 4$ Butler matrix for wireless communications.

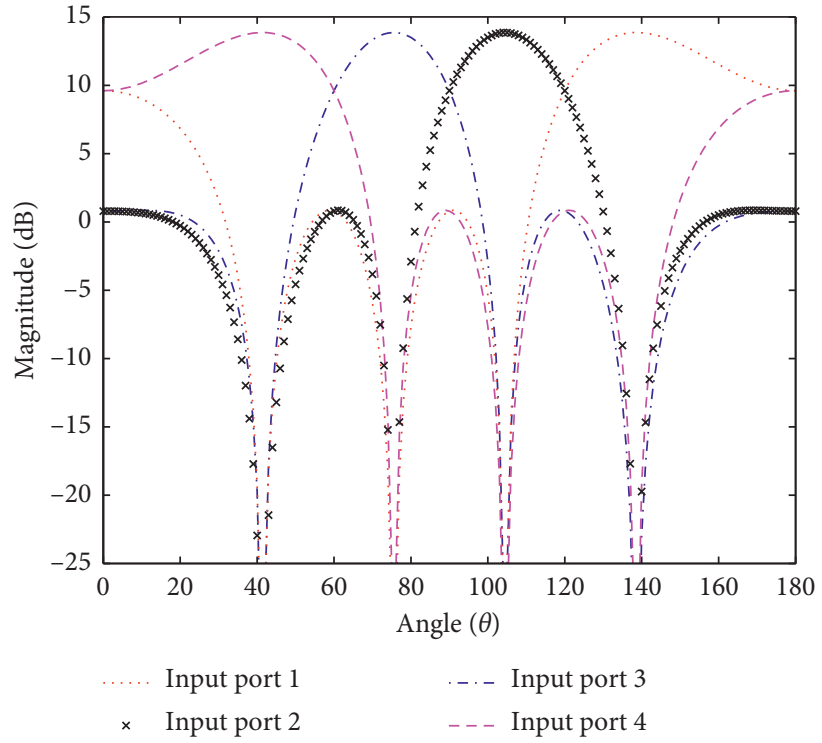

(a)

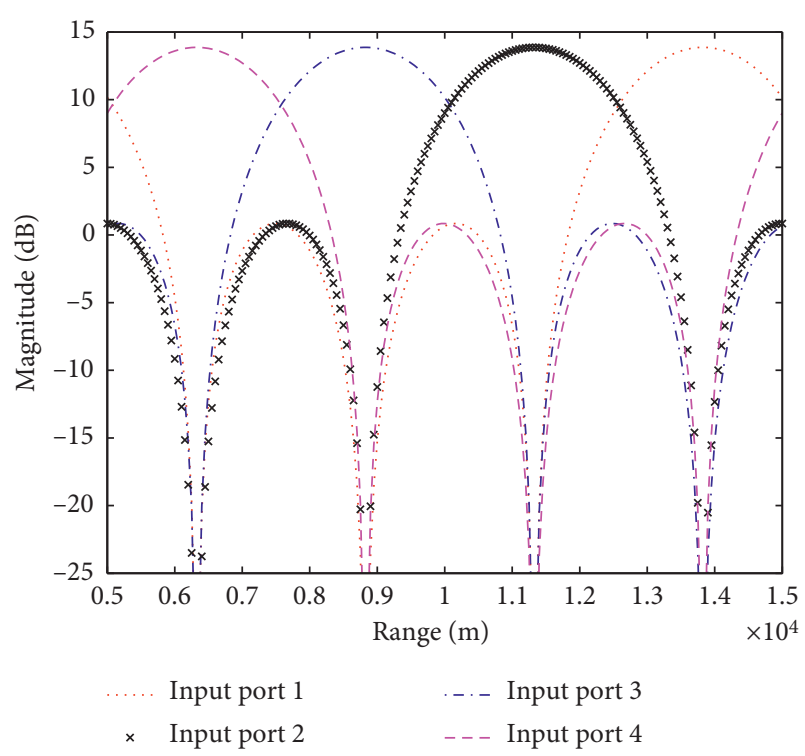

(b)

Figure 10: FDA $4 \times 4$ Butler matrix input ports excited radiation power pattern when $\Delta f=30 \mathrm{kHz}$ is adopted (a) in angle dimension and (b) in range dimension.

In Figure 10, we simulate the far-field radiation power pattern in angle and range dimensions for each of the four input port excitations, namely, "1," "2," " 3 ," and "4," respectively. It is evident that the four main beams are orthogonal to each other, pointing along $41^{\circ}, 76^{\circ}, 104^{\circ}$, and $139^{\circ}$, respectively, in angle dimension (Figure 10(a)), whereas in range dimension (Figure 10(b)), the beams point along $6.2 \mathrm{~km}, 8.9 \mathrm{~km}, 11.2 \mathrm{~km}$, and $13.9 \mathrm{~km}$, respectively.

\section{Performance Analysis of FDA Antenna}

6.1. Signal-to-Interference-and-Noise Ratio (SINR). In this section, we discuss the output SINR of the FDA antenna beamformer with respect to its spatial resolution (mainlobe) and interference rejection capability (sidelobe level and spectral nulls). According to [19], the output SINR can be expressed as

$$
\operatorname{SINR} \triangleq \frac{\sigma_{s}^{2}\left|\mathbf{w}^{H} \mathbf{a}(t-\tau, \theta, r)\right|^{2}}{\mathbf{w}^{H}\left(\sum_{i} \sigma_{i}^{2} \mathbf{a}\left(t-\tau, \theta_{i}, r_{i}\right) \mathbf{a}^{H}\left(t-\tau, \theta_{i}, r_{i}\right)+\sigma_{n}^{2}\right) \mathbf{w}},
$$

where $\sigma_{s}^{2}$ denotes the variance of the intended user/target signal. In case, the user (target) is being observed in the background of few weak interferences, (16) is simplified to (17):

$$
\operatorname{SINR} \simeq \frac{\sigma_{s}^{2} M^{2}}{\sigma_{n}^{2}} .
$$

On the contrary, if the user (target) is observed in the background of strong interferences, then we have 


$$
\mathrm{SINR} \simeq \frac{\sigma_{s}^{2} M^{2}}{\sum_{i} \sigma_{i}^{2}\left|\mathbf{w}^{H} \mathbf{a}\left(t-\tau, \theta_{i}, r_{i}\right)\right|^{2}\left|\mathbf{w}^{H} \mathbf{a}\left(t-\tau, \theta_{i}, r_{i}\right)\right|^{2}}
$$

6.2. Direction of Arrival (DOA). In array signal processing, direction of arrival (DOA) estimation, also known as angle of arrival (AOA) detection is an important concept [47]. The main objective of direction of arrival (DOA) estimation is to utilize the data received on the uplink at the base-station antenna array in order to estimate the directions of the signals from the desired mobile users and the directions of interference signals. For instance, suppose several transmitters are employed simultaneously to work, hence each source will process the potential multipath components at the receivers. Therefore, it is essential for the receiving antennas to able to estimate those angles of arrival to know the possible location of transmitters and/or users.

In this paper, we resort to the classical DOA algorithm, namely, multiple signal classification (MUSIC) to resolve multipath signals DOA estimation. The MUSIC algorithm employs the covariance matrix of the received data to separate the signal subspace and noise subspace. And then it creates space scanning spectrum to search the peaks in the whole region by utilizing orthogonality between the signal directional vector and noise subspace, in order to estimate signals' DOA [47]. The MUSIC for FDA antenna can be expressed as

$$
P(\theta, r)=\frac{1}{\mathbf{a}_{r}^{H}(\theta, r) \mathbf{V}_{n} \mathbf{V}_{n}^{H} \mathbf{a}_{r}(\theta, r)},
$$

where $\mathbf{V}_{n}$ denotes the noise subspace and $\mathbf{a}_{r}(\theta, r)$ is given as

$$
\mathbf{a}_{r}(\theta, r)=\left[1 \exp \left(j \psi_{r_{1}}\right) \cdots \exp \left(j \psi_{r_{n}}\right) \cdots \exp \left(j \psi_{r_{N-1}}\right)\right]
$$

with $\psi_{r_{n}}$ being expressed as

$$
\psi_{r_{n}}=2 \pi\left(f_{0} \frac{n d \sin \theta}{c}+\frac{\Delta F_{n} r}{c}+\frac{n \Delta F_{n} d \sin \theta}{c}\right) .
$$

\section{Numerical Results}

In this section, we use (17) and (18) to show the results of SINR as a function of SNR. Figure 11 depicts comparative performance of FDA antenna and phased array antenna in noise dominant. It can be seen that both antenna systems exhibited the same performance.

But in Figure 12, the FDA antenna shows a better SINR performance compared to phased array antenna because the FDA antenna has the capability to suppress rangedependent interferences, especially, where there are strong interferences with angle being the same but different range form the intended user.

Finally, we assume the following parameters: $M=10$, $\mathrm{SNR}=10$, and number of snapshots $=100$. Figure 13(a), shows the DOA performance between the FDA antenna and

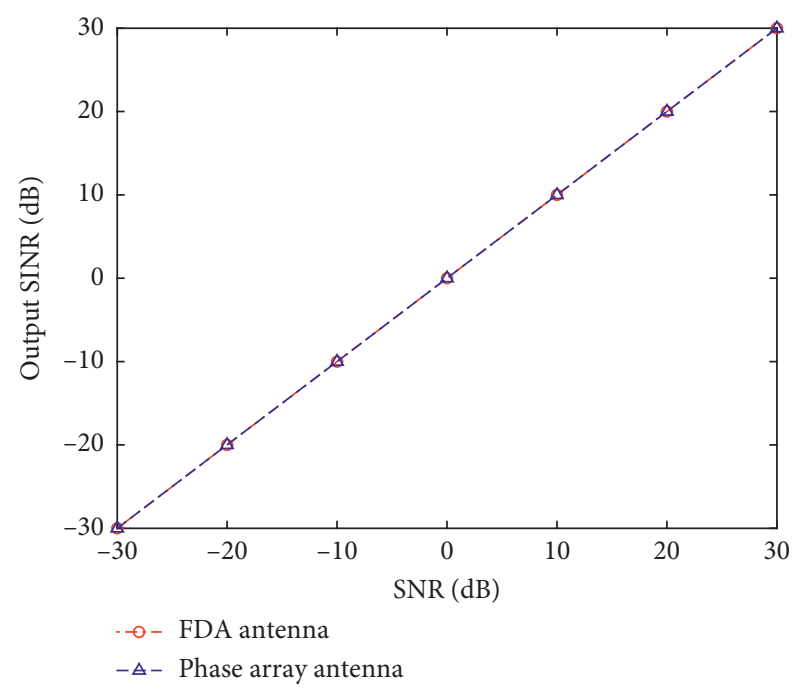

FIGURE 11: SINR as a function of SNR in noise dominant.

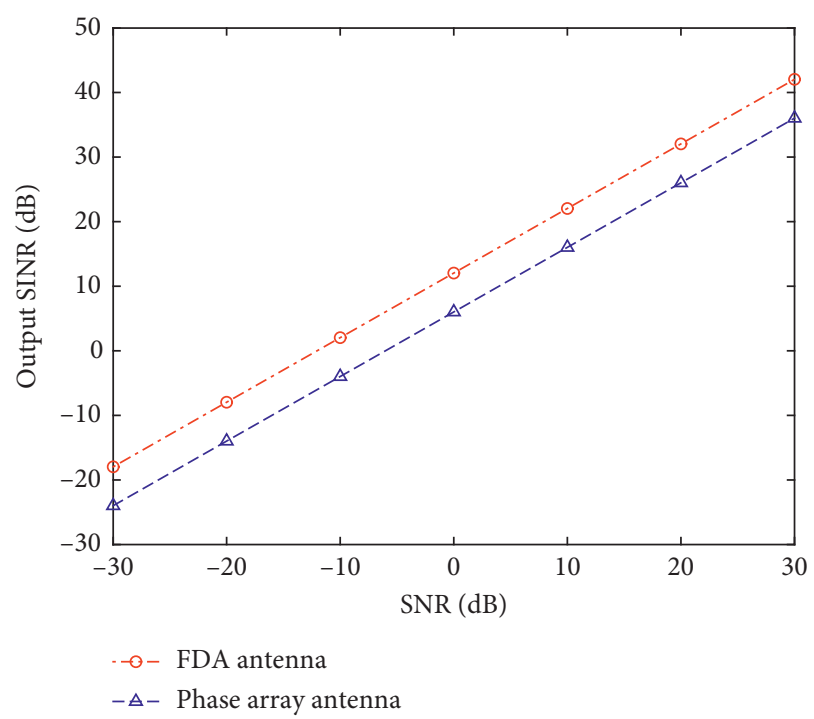

FIGURE 12: SINR as a function of SNR in interference dominant.

phased array antenna in angle dimension. It can be observed that both FDA antenna and phased array antenna have the same DOA performances towards angle directions, such as $\left[40^{\circ} 60^{\circ}\right]$. In Figure $13(\mathrm{~b})$, that is range dimension, phased array antenna lack range resolution. In contrast, the FDA antenna still has DOA performance towards the range directions, such as $8 \mathrm{~km}$ and $10 \mathrm{~km}$ which is unavailable to phased array antenna. Therefore, the FDA antenna has range-dependent capability, and it can effectively identify signal sources.

7.1. Hardware/Software Complexity Discussions. Antenna Configuration: Apart from linear array antenna configuration, different antenna geometry can be adopted in designing FDA such as planar and circular. In phased array antenna, the cost of using phase shifters is very high. In FDA, no phase shifters are required. The most important difference of FDA 


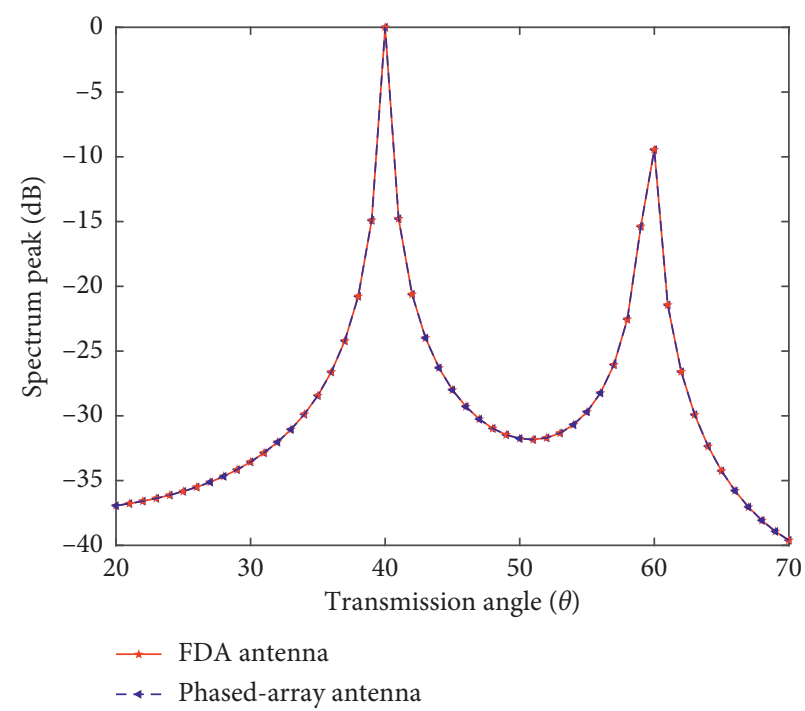

(a)

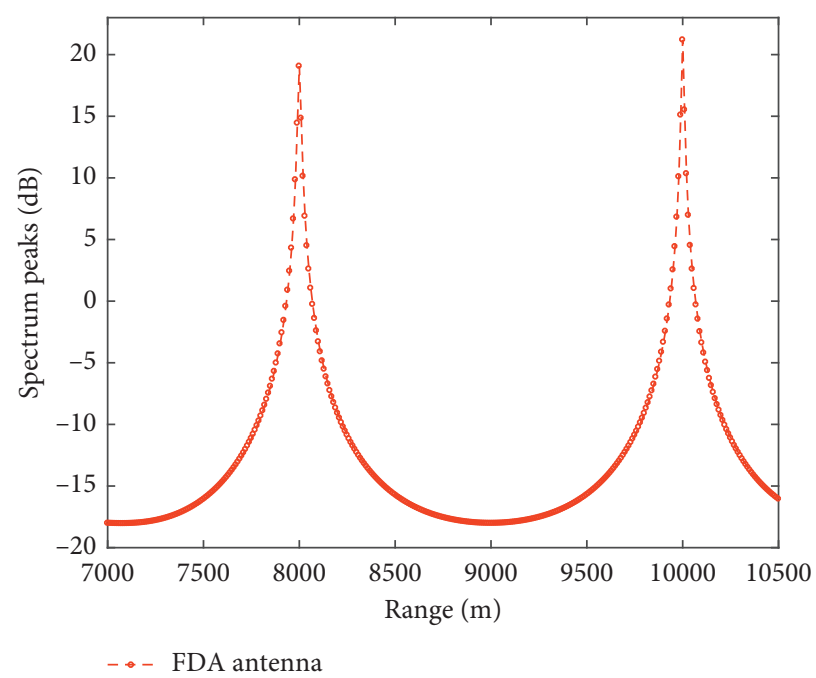

(b)

FIGURE 13: MUSIC pseudospectra: (a) in angle dimension and (b) in range dimension.

antenna from a phased array antenna is that a small amount of frequency increment compared to the carrier frequency is used across the array elements.

Hardware/software complexity: FDA with constant element spacing may not be an ideal configuration due to the frequency diversity. We can employ larger element spacing to reduce the array complexity. Implementation of FDA concept on array hardware may require agile local oscillators and mixers to change the frequency for each element of the array. The complex transmit-receive weighting functions of FDA depends on the baseband frequency and frequency increment. We can relax this weighting function's frequency increment dependence. This means that spatial weighting can be factored out from the summation and applied once to the entire signal, thus reducing computation complexity.

\section{Conclusion}

In this paper, we have shown the capability of FDA antenna from its origin to the potential applications in wireless communication systems. The range-angle-dependent array factor permits the FDA antenna to transmit energy over a desired range dimension and angle dimension. Because of the range-angle-dependent property, the FDA has the potential to suppress range-dependent interference. Thus, this provides an extra degree of freedom unavailable to the phased array antennas. In addition, we have discussed application scenario of using FDA and Butler matrix for communication. Numerical example shows that the FDA antenna is a promising array concept for wireless communication systems. Certainly, there may be more problems to be investigated by employing FDA antenna in communication applications. We also call for more support on the research and development of FDA antenna for future wireless communication systems.

\section{Conflicts of Interest}

The authors declare that there are no conflicts of interest regarding the publication of this paper.

\section{References}

[1] N. N. Alotaibi and K. A. Hamdi, "Switched phased-array transmission architecture for secure millimeter-wave wireless communication," IEEE Transactions On Communications, vol. 64, no. 3, pp. 1303-1312, 2016.

[2] W. Roh, J.-Y. Seol, J. Park et al., "Millimeter-wave beamforming as an enabling technology for $5 \mathrm{~g}$ cellular communications: theoretical feasibility and prototype results," IEEE Communications Magazine, vol. 52, no. 2, pp. 106-113, 2014.

[3] L. C. Godara, "Applications of antenna arrays to mobile communications, part I: performance improvement, feasibility, and system considerations," Proceedings of the IEEE, vol. 85, no. 7, pp. 1031-1060, 1997.

[4] L. C. Godara, "Application of antenna arrays to mobile communications, part II: beam-forming and direction-ofarrival considerations," Proceedings of the IEEE, vol. 85, no. 8, pp. 1195-1245, 1997.

[5] P. F. McManamon, P. J. Bos, M. J. Escuti et al., "A review of phased array steering for narrow-band electrooptical systems," Proceedings of the IEEE, vol. 97, no. 6, pp. 1078-1096, 2009.

[6] J. Li and P. Stoica, "The phased array is the maximum SNR active array," IEEE Signal Processing Magazine, vol. 27, no. 2, pp. 143-144, 2010.

[7] E. Brookner, "Phased array radars-past, present and future," in 2002 International Radar Conference (Radar 2002), pp. 104-113, Santa Barbara, CA, USA, April 2002.

[8] J. H. G. Ender, H. Wilden, U. Nickel et al., "Progress in phasedarray radar applications," in Proceedings of the IEEE First European Radar Conference, EURAD, pp. 113-116, October 2004.

[9] P. Antonik, An investigation of a frequency diverse array, Ph.D. dissertation, University College London, London, UK, 2009. 
[10] P. Antonik, M. C. Wicks, H. D. Griffiths et al., "Frequency diverse array radars," in 2006 IEEE Conference on Radar, pp. 215-217, Verona, NY, USA, April 2006.

[11] W. Q. Wang and J. Cai, "MIMO SAR using chirp diverse waveform for wideswath remote sensing," IEEE Transactions on Aerospace and Electronic Systems, vol. 48, no. 4, pp. 3171-3185, 2012.

[12] A. M. Haimovich, R. Blum, and L. Cimini, "MIMO radar with widely separated antennas," IEEE Signal Processing Magazine, vol. 25, no. 1, pp. 116-129, 2008.

[13] J. Li and P. Stoica, "MIMO radar with colocated antennas," IEEE Signal Processing Magazine, vol. 24, no. 5, pp. 106-114, 2007.

[14] Y. Ding, J. Zhang, and V. Fusco, "Frequency diverse array OFDM transmitter for secure wireless communication," Electronics Letters, vol. 51, no. 17, pp. 1374-1376, 2015.

[15] W. Q. Wang, "Mitigating range ambiguities in high-PRF SAR with OFDM waveform diversity," IEEE Geoscience and Remote Sensing Letters, vol. 10, no. 1, pp. 101-105, 2013.

[16] F. S. Johansson, L. G. Josefsson, and T. Lorentzon, "A novel frequency-scanned reflector antenna," IEEE Transactions on Antennas and Propagatio, vol. 37, no. 8, pp. 984-989, 1989.

[17] C. Vazquez, C. Garcia, Y. Alvarez et al., "Near field characterization of an imaging system based on a frequency scanning antenna array," IEEE Transactions on Antennas and Propagation IEEE Transactions on Antennas and Propagation, vol. 61, no. 5, pp. 2874-2879, 2013.

[18] W. Q. Wang, "Range-angle dependent transmit beampattern synthesis for linear frequency diverse arrays," IEEE Transactions on Antennas and Propagation, vol. 61, no. 8, pp. 4073-4081, 2014.

[19] W. Q. Wang, "Overview of frequency diverse array in radar and navigation applications," IET Radar, Sonar and Navigation, vol. 10, no. 6, pp. 1001-1012, 2016.

[20] H. Shao, J. Li, H. Chen et al., "Adaptive frequency offset selection in frequency diverse array radar," IEEE Antennas and Wireless Propagation Letters, vol. 13, no. 1, pp. 1405-1408, 2014.

[21] W. Q. Wang and H. C. So, "Transmit subaperturing for range and angle estimation in frequency diverse array radar," IEEE Transactions on Signal Processing, vol. 62, no. 8, pp. 20002011, 2014.

[22] Y. Wang, W. Q. Wang, H. Chen et al., "Optimal frequency diverse subarray design with Cramer-Rao lower bound minimization," IEEE Antennas and Wireless Propagation Letters, vol. 14, no. 1, pp. 1188-1191, 2015.

[23] B. Abdul, W. Khan, S. Khan, and I. M. Qureshi, "Development of frequency diverse array radar technology: a review," IET Radar, Sonar and Navigation, vol. 12, no. 2, pp. 165-175, 2018.

[24] J. Xiong, S. Y. Nusenu, and W. Q. Wang, "Directional modulation using frequency diverse array for secure communications," Wireless Personal Communications, vol. 95, no. 3, pp. 2979-2689, 2017.

[25] J. Hu, S. Yan, F. Shu et al., "Artificial-noise-aided secure transmission with directional modulation based on random frequency diverse arrays," IEEE Access, vol. 5, pp. 1658-1667, 2017.

[26] S. Y. Nusenu, W. Q. Wang, and J. Xiong, "Time-modulated frequency diverse array for physical-layer security," IET Microwaves Antenna and Propagation, vol. 11, no. 9, pp. 1274-1279, 2017.

[27] S. Y. Nusenu, W.-Q. Wang, and S. Ji, "Secure directional modulation using frequency diverse array antenna," in 2017 IEEE Radar Conference, pp. 378-382, Seattle, WA, USA, May 2017.
[28] W. Q. Wang, "DM using FDA antenna for secure transmission," IET Microwaves Antenna and Propagation, vol. 11, no. 3, pp. 336-345, 2017.

[29] L. Zhuang and X. Z. Liu, "Precisely beam steering for frequency diverse arrays based on frequency offset selection," in IEEE International Radar Conference, pp. 1-4, Bordeaux, France, 2009.

[30] W. Khan, I. M. Qureshi, and S. Sarah, "Frequency diverse array radar with logarithmically increasing frequency offset," IEEE Antennas And Wireless Propagation Letters, vol. 14, pp. 499-502, 2015.

[31] B. Abdul, I. M. Qureshi, W. Khan et al., "Beam pattern synthesis for an FDA radar with Hamming window-based nonuniform frequency offset," IEEE Antennas and Wireless Propagation Letters, vol. 16, pp. 2283-2286, 2017.

[32] A. Mukherjee, S. A. A. Fakoorian, J. Huang, and A. L. Swindlehurst, "Principles of physical layer security in multiuser wireless networks: a survey," IEEE Communications Surveys and Tutorials, vol. 16, no. 3, pp. 1550-1573, 2014.

[33] A. D. Wyner, "The wire-tap channel," Bell System Technical Journal, vol. 54, no. 8, pp. 1355-1387, 1975.

[34] P. K. Gopala, L. Lai, and H. El-Gamal, "On the secrecy capacity of fading channels," IEEE Transactions on Information Theory, vol. 54, no. 10, pp. 4687-4698, 2008.

[35] H.-M. Wang, M. Luo, Q. Yin, and X.-G. Xia, "Hybrid cooperative beamforming and jamming for physical-layer security of two-way relay networks," IEEE Transactions on Information Forensics and Security, vol. 8, no. 12, pp. 20072020, 2013.

[36] N. Valliappan, A. Lozano, and R. W. Heath, “Antenna subset modulation for secure millimeter-wave wireless communication," IEEE Transactions on Communications, vol. 61, no. 8, pp. 3231-3245, 2013.

[37] J. Kim, A. Ikhlef, and R. Schober, "Combined relay selection and cooperative beamforming for physical layer security," Journal of Communications and Networks, vol. 14, no. 4, pp. 364-373, 2012.

[38] H. Ma and P. Ma, "Beamforming design of decode-andforward cooperation for improving wireless physical layer security," in 15th International Conference on Advanced Communications Technology, pp. 41-49, Pyeongchang, Republic of Korea, January 2013.

[39] C.A. Balanis, Antenna Theory Analysis and Design, John Wiley and Sons, New York, NY, USA, 1997.

[40] T. Eker, S. Demir, and A. Hizal, "Exploitation of linear frequency modulated continuous waveform (LFMCW) for frequency diverse arrays," IEEE Transactions on Antennas and Propagation, vol. 61, no. 7, pp. 3546-3553, 2013.

[41] A. Jones and B. Rigling, "Planar frequency diverse array receiver architecture," in 2012 IEEE Radar Conference, pp. 145-150, Atlanta, GA, USA, May 2012.

[42] M. Campo, W. Simon, and R. Baggen, "Steerable antenna array at $24 \mathrm{GHz}$ using Butler matrices and MEMS-switches," in Proceedings of the IEEE International Symposium on Antennas and Propagation, pp. 1-2, Kamp-Lintfort, Germany, July 2012.

[43] W. Bhowmik and S. Srivastava, "Optimum design of a 4x4 planar Butler matrix array for WLAN application," Journal of Telecommunications, vol. 2, no. 1, pp. 68-74, 2010.

[44] M. Ueno, "A systematic design formulation for Butler matrix applied FFT algorithm," IEEE Transactions on Antennas and Propagation, vol. 29, no. 3, pp. 496-501, 1981.

[45] R. J. Mailloux, Phased Array Antenna Handbook, Artech House Inc., Boston, MA, USA, 2005. 
[46] Y. Ding and V. Fusco, "Sidelobe manipulation using Butler matrix for $60 \mathrm{GHz}$ physical layer secure wireless communication," in 2013 Loughborough Antennas and Propagation Conference, pp. 61-65, Loughborough, UK, November 2013.

[47] F. B. Gross, Smart Antenna for Wireless Communications: With Matlab, McGraw-Hill Companies, New York, NY, USA, 1st edition, 2005. 


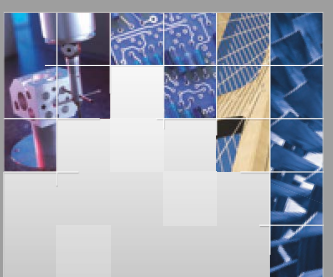

\section{Enfincering}
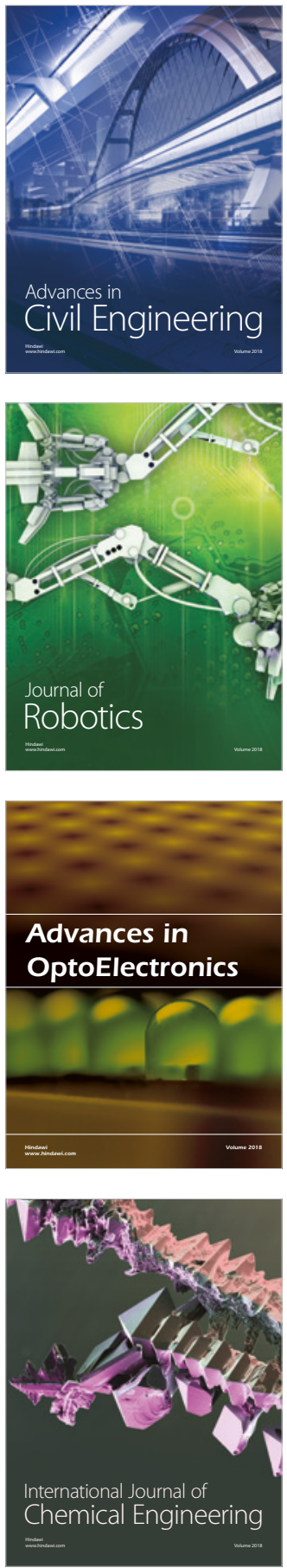

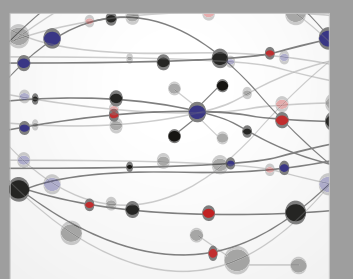

\section{Rotating \\ Machinery}

The Scientific World Journal

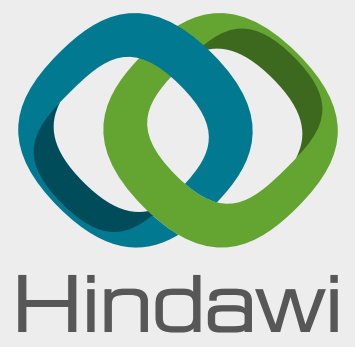

Submit your manuscripts at

www.hindawi.com
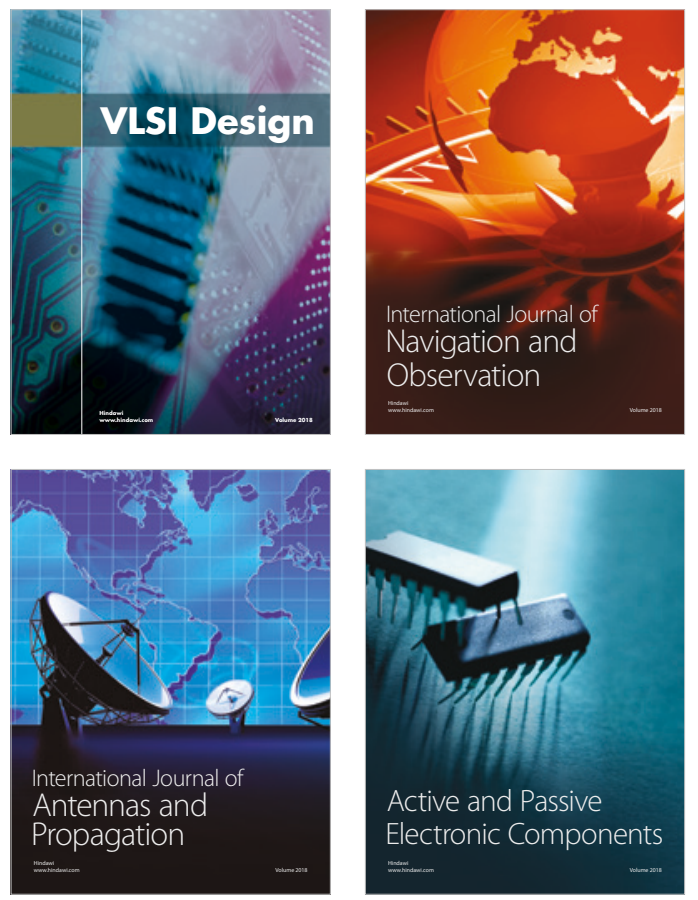
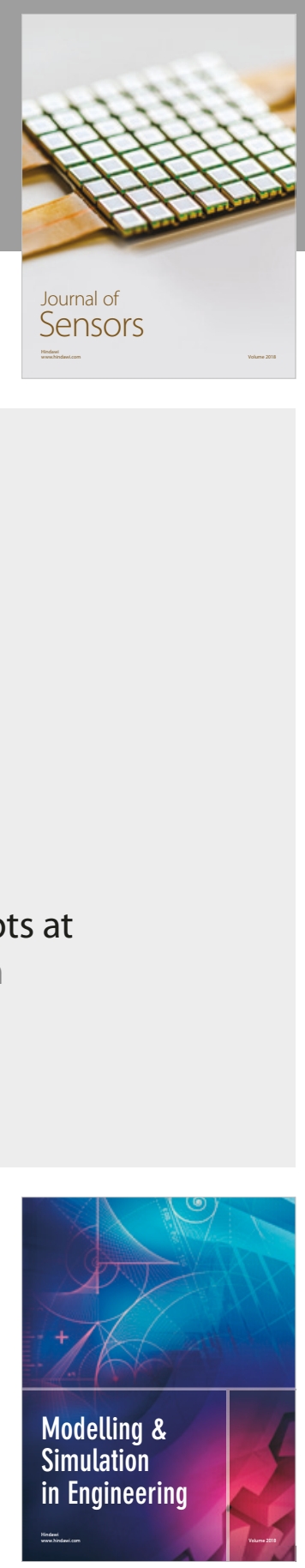

\section{Advances \\ Multimedia}
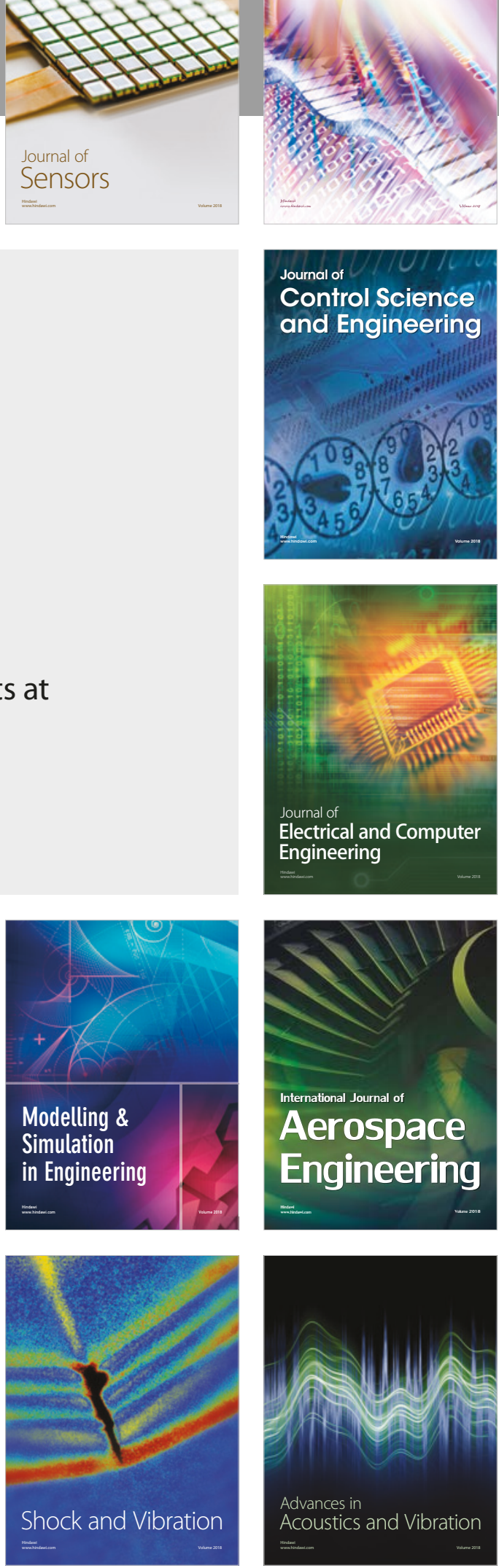